\title{
http://bjas.journals.ekb.eg \\ Intrathecal Drug Therapy in Chronic Pain Management
}

\author{
E.A.Hanafy, E.E. Mostafa and S.M.Hussein
}

Anesthesiology and Intensive Care Unit and Pain Management Dept., Faculty of Medicine, Benha Univ., Benha, Egypt

E-Mail:Shimaa2@gmail.com

\begin{abstract}
Chronic pain impacts the quality of multiple functional domains, including family life, workplace performance, social interactions, and sleep patterns. The management of chronic pain continues to pose many challenges to healthcare providers. Intrathecal drug delivery systems (IDDS) provide an effective therapy for patients suffering from chronic pain intractable to medical management. to identify best practices and provide guidance to clinicians to ensure safety and optimize intrathecal drug delivery for chronic intractable pain management. This is a review article, The search was performed in MEDLINE, Embase, Pubmed and CINAHL Plus in the same date range with the following mediacl terms. Intrathecal drug (IT) delivery has many benefits compared with oral or parenteral routes for pain control. First, it allows for the use of significantly lesser doses of opioids. Second, it bypasses the first pass effect and the blood brain barrier. Third, patients typically experience fewer adverse effects (AEs) due to the decreased total dose of opioid used. However, IT therapy may be associated with complications related to many factors, including those related to the IT pump itself (e.g., mechanical failure), pump pocket fills, issues surrounding preparation of the IT medication and programming of the pump for medication release, and complications following pump implantation surgery. Optimal use of this therapy depends on a thorough understanding of intrathecal physiology and anatomy as well appreciation of physicochemical properties of administered medications and the mode of IT delivery.
\end{abstract}

Keywords: Intrathecal, Chronic pain, Opioids.

\section{Introduction}

The delivery of drugs into the subarachnoid space dates back to the late 19th century when August Bier reported using intrathecal (IT) cocaine on himself and several patients undergoing lower extremity surgical procedures [1].

This was followed by the introduction of opioids (morphine) into the subarachnoid space by Rudolph Matas and Otojiro Kitagawa in 1900 and 1901, respectively. These initial publications spawned research into the field of neuraxial anesthesia, and it was not until the 1940s when IT drug delivery moved beyond single shot injections and continuous IT drug delivery was first introduced in animal models [2].

In the 1970s, spinal opioid receptors were first discovered, giving more credence to the field of IT opioid administration for the treatment of pain, and leading to further advancements in its clinical utility. The first report of using IT morphine for intractable cancer pain in the form of single shot injections was in 1979. Then, in 1981 Onofrio and colleagues published the first use of an implantable intrathecal drug delivery system (IDDS) to treat cancer pain. In 1983, Coombs etal published an article describing IT morphine use in an implantable reservoir in a group of 10 patients with cancer and non cancer related pain . This was followed by several more studies describing its use in treating cancer and non cancer pain. [3]

Since these original studies, IT pump therapy has evolved significantly, with improvements in technology, drug selection, and patient procedural techniques. Intrathecal drug delivery has many benefits compared with oral or parenteral routes for pain control. First, it allows for the use of significantly lesser doses of opioids compared with the oral or parenteral routes for similar efficacy given that medications are deposited directly at the site of spinal action. Second, it bypasses the first pass effect and the blood brain barrier. Third, patients typically experience fewer adverse effects (AEs) due to the decreased total dose of opioid used. Fourth, other analgesics may be used in addition to opioids for improved pain control. However, in order to take advantage of this, proper patient selection and careful application of the therapy needs to be exercised. Nonetheless, there are unique complications to chronic IT drug delivery that need to be taken into consideration. Optimal use of this therapy depends on a thorough understanding of intrathecal physiology and anatomy as well appreciation of physicochemical properties of administered medications and the mode of IT delivery . [1]

The objective of this study was to identify best practices and provide guidance to clinicians to ensure safety and optimize intrathecal drug delivery for chronic intractable pain management.

\section{Methods}

This is a review article, The search was performed in MEDLINE, Embase, Pubmed and CINAHL Plus in the same date range with the following mediacl terms: "Intrathecal; chronic pain; opioids", including articles from 2000 to 2019, Excluded articles from review are those of language other than English.

\section{Results}

Chronic pain is a major burden and an expensive economic impact and a public health problem in 
industrialized countries. Chronic pain impacts the quality of multiple functional domains, including family life, workplace performance, social interactions, and sleep patterns. Chronic pain can be cancer related and noncancer related, with the latter accounting for the vast majority of patients. Pain of spinal origin, and in particular chronic low back pain, is foremost in prevalence and significant among chronic non-cancer pain.

The management of chronic pain continues to pose many challenges to healthcare providers. Intrathecal drug delivery systems (IDDS) provide an effective therapy for patients suffering from chronic pain intractable to medical management.An intrathecal drug delivery system is composed of a surgically implanted battery-operated pump and medication reservoir connected to an intrathecal catheter. The tip of the catheter is placed into the CSF.
The traditional teaching of many instructors has reasonably recommended placing the catheter tip close to the target receptors of the spinal segment(s) associated with the dermatome/sclerotome/ viscerotome of the primary pain generator. This issue of delivery location reflects on the fact that analgesic medications (mu opioid, alpha 2 adrenergic, $\mathrm{N}$ type calcium channel blockers), aside from the local anesthetics, exert their effects on the target receptors/channels that are located on the terminals of the primary afferent and at the level of the first order spinal synapse; and the need for the drug to reach the spinal levels associated with the spinal segments processing the pain information (where the target receptors are located), and the absence of robust infusate redistribution. The ability to determine this location is, for the most part, difficult, and may be easier to determine when local anesthetics are administered Table (1).

Table (1) Intrathecal Drug Delivery Systems [4].

\begin{tabular}{|c|c|c|c|c|}
\hline & Codman 3000 & $\begin{array}{l}\text { Medtronic } \\
\text { Isomed }\end{array}$ & $\begin{array}{c}\text { Flowonix } \\
\text { Prometra II }\end{array}$ & $\begin{array}{c}\text { Medtronic } \\
\text { SynchroMed II }\end{array}$ \\
\hline Catheter material & $\begin{array}{l}\text { Polyurethane } \\
\text { with titanium } \\
\text { reinforced coil }\end{array}$ & $\begin{array}{c}\text { Radiopaque } \\
\text { silicone rubber } \\
\text { with titanium tip }\end{array}$ & $\begin{array}{c}\text { Radiopaque } \\
\text { silicone rubber } \\
\text { with tungsten tip }\end{array}$ & $\begin{array}{l}\text { Radiopaque silicone } \\
\text { rubber with titanium } \\
\text { tip }\end{array}$ \\
\hline Pump material & $\begin{array}{c}\text { Titanium/silicone } \\
\text { rubber }\end{array}$ & $\begin{array}{c}\text { Titanium/silicone } \\
\text { rubber }\end{array}$ & $\begin{array}{c}\text { Titanium/silicone } \\
\text { rubber }\end{array}$ & $\begin{array}{c}\text { Titanium/silicone } \\
\text { rubber }\end{array}$ \\
\hline Pump mechanics & $\begin{array}{c}\text { Continuous flow } \\
\text { propellant }\end{array}$ & $\begin{array}{c}\text { Continuous flow } \\
\text { propellant }\end{array}$ & $\begin{array}{c}\text { Valve gated } \\
\text { programmable }\end{array}$ & $\begin{array}{c}\text { Peristaltic titanium/ } \\
\text { plastic } \\
\text { programmable }\end{array}$ \\
\hline MRI compatibility & No effects $3 \mathrm{~T}$ & No effects $1.5 \mathrm{~T}$ & $\begin{array}{l}\text { MRI conditional } \\
1.5 \mathrm{~T} \text { with valve } \\
\text { shut-off }\end{array}$ & MRI conditional 3T \\
\hline $\begin{array}{l}\text { Patient-controlled } \\
\text { intrathecal analgesia } \\
\text { (PCITA) }\end{array}$ & None & None & $\begin{array}{l}\text { Patient therapy } \\
\text { controller (PTC) }\end{array}$ & $\begin{array}{l}\text { Personal therapy } \\
\text { manager (PTM) }\end{array}$ \\
\hline
\end{tabular}

It is far less clear when baclofen is infused to treat spasticity or morphine to relieve pain. An important issue is the appreciation that the receptors associated with the target dermatome are not restricted to the spinal segment associated with the root dermatome. Afferent input into any given segment may send collaterals up to several segments rostrally and caudally. It has been argued that the IT drug must accordingly reach the cells and afferent terminals in these distal dermatomes Intrathecal drug delivery has many benefits compared with oral or parenteral routes for pain control. First, it allows for the use of significantly lesser doses of opioids compared with the oral or parenteral routes for similar efficacy given that medications are deposited directly at the site of spinal action. Second, it bypasses the first pass effect and the blood brain barrier. Third, patients typically experience fewer adverse effects (AEs) due to the decreased total dose of opioid used. Fourth, other analgesics may be used in addition to opioids for improved pain control. However, in order to take advantage of this, proper patient selection and careful application of the therapy needs to be exercised. Nonetheless, there are unique complications to chronic IT drug delivery that need to be taken into consideration.

The main indications for intrathecal drug therapy in chronic pain (benign and cancer-related) are neuropathic pain, postherpetic neuralgia, peripheral neuropathy, and mixed nociceptiveneuropathic pain syndromes. Such syndromes include failed back surgery syndrome (FBSS), type I nociceptive pain, visceral pain including chronic pancreatitis, insufficient pain relief, severe side effects to oral, rectal, or transdermal opioid administration. This technique is also applied in patients with severe spasticity.

Optimal use of this therapy depends on a thorough understanding of intrathecal physiology 
and anatomy as well appreciation of physicochemical properties of administered medications and the mode of IT delivery

IT therapy may be associated with complications related to many factors, including those related to the IT pump itself (e.g., mechanical failure), pump pocket fills, issues surrounding preparation of the IT medication and programming of the pump for medication release, and complications following pump implantation surgery

Morphine and ziconotide are the only IT agents approved by the Food and Drug Administration (FDA) for the management of chronic pain. However, a number of other medications including other opioids (hydromorphone and fentanyl), the local anesthetic bupivacaine, and the alpha-2 adrenergic agonist clonidine are often used as standard of care IT agents. Of note, IT baclofen is FDA approved for the management of spasticity.

\section{Discussion}

Intrathecal administration of analgesics is only indicated in patients with persistent pain in whom conventional methods of pain management have failed. These methods include extensive pharmacological therapy (analgesics according to the 3 -step analgesic ladder including opioid rotation, if indicated combined with antidepressants, antiepileptic drugs, topical treatment and potentially also ketamine), interventional pain management techniques, rehabilitation, and cognitive and behavioral therapy. There should be no (new) surgical indication. As a result, intrathecal drug administration comes at the end of the treatment ladder of patients with chronic noncancer pain. Especially in cases of chronic, noncancer pain syndromes, patients should first undergo extensive psychological evaluation [5].

The presence of psychological factors such as psychopathology, mood disorders, severe distress, and functional pain syndromes (somatization) reportedly reduce the chance of a successful treatment. Untreated addiction is an absolute contraindication for this form of therapy. The pathophysiological mechanism of the pain syndrome also affects the results of the treatment. The chance of effective pain relief is significantly lower when opioids alone are administered for the treatment of patients with neuropathic pain compared with patients with nociceptive or mixed (nociceptiveneuropathic) pain [6].

This means that every patient candidate should be subjected to extensive multidisciplinary evaluation before the therapy is initiated. This could include, for example, an anesthesiologist and/or neurologist and/or neurosurgeon to evaluate the pain syndrome and a psychologist and/or psychiatrist to evaluate the psychosocial context and to exclude a psychiatric diagnosis. For the treatment of chronic noncancer pain, it is essential to begin with a trial period using a temporary intrathecal pain pump to evaluate the patient's response before a definite intrathecal pain pump is implanted [6].

The test period can be evaluated (positive if the treatment induces effective pain relief) by means of the following arbitrary objectives: pain relief of at least 50\%; significant improvement of the quality of life and sleep; significant increase in daily activities; and pain medication (nonintrathecal) can be reduced or entirely discontinued. Further studies are needed to establish if these objectives during the trial period have a predictive value for the effectiveness of pain relief after implantation of the pain pump. The polyanalgesic consensus conference suggested a single-shot trial in case of nociceptive pain. Trialing the effect of ITDD for mixed pain or neuropathic pain, continuous infusion over several days may be preferred if the patient tolerates prolonged infusion [7].

In patients with pain due to cancer, intrathecal drug administration can be considered when oral or transdermal analgesics provide insufficient pain relief or produce unacceptable side effects. In these patients, the intrathecally administered drugs may be combinations of more than 1 agent.Patients with cancer-related pain who are candidates for intrathecal drug administration may have a short life expectancy. In these cases, the switch from oral or transdermal to intrathecal drug administration may be accomplished without a trial period, especially given that implantation of the drug administration pump is rarely performed. Patients are implanted with a subcutaneous port and infused intrathecally with an external pump[8].

Complications of IDDS can be categorized based as pharmacologic (covered in "Intrathecal Agents" section), procedural, equipment, or programming related. Catheter malfunction tends to be the most common type of complication associated with IDDS hardware and more recent studies report an incidence between $15 \%-25 \%$. These complications include occlusion, leakage, migration, dislodgement, kinking, puncture or shearing of the catheter at various sites from the pump to the CSF. Catheter malfunction is not only an inconvenience to the patient and physician, but also puts the patient at risk for uncontrolled pain and opioid withdrawal from abrupt cessation of IT drug delivery and exposing them to the additive risks of additional invasive procedures. Altogether, this decreases the cost-effectiveness of IDDS. Shearing of catheters is more common when the midline approach is used due to the proximity of the spinous processes particularly with thoracolumbar extension. For this reason, the paramedian approach to insertion of IT catheters is common practice [9].

In addition to proper surgical technique, type of catheter used may play a role, and several new catheters have been designed to help reduce these complication rates. Procedural related complications 
include postdural puncture headaches (PDPHs), hematomas, spinal cord injury, wound dehiscence or skin erosion, CSF leaks, and patient discomfort at the site of the pump reservoir. Patient positioning in a supine position for 24 hours postoperatively is suggested to reduce the risk of PDPHs [4].

If a PDPH is unresponsive to conservative medical management, an epidural blood patch can be performed. Spinal cord injury can be avoided by entering the IT space at L2-3 or L3-4 level. Placement of an abdominal binder postoperatively can help reduce the risk of hematoma formation at the pump reservoir site. However, the majority of these risks can be avoided with good surgical technique. An often underappreciated complication is that of subcutaneous drug administration during IDDS refills, also known as pocket fill. This complication can seemingly go unnoticed if inexperienced personnel administer these refills with potential for overdose from a bolus of concentrated medication administered acutely in the subcutaneous tissue. Therefore, it is important to recognize this risk and ensure that pump refills only be performed by properly trained and experienced personnel. The rate of IDDS related infections varies in the literature from $0.5 \%-9 \%$, and most commonly involves superficial wound infections with Staphylococcus species. However, vigilance to prevent and detect infections early is still important to prevent more serious infections such as meningitis, which is rare (1\%) but still significant. Use of proper sterile technique, perioperative antibiotics (cephalosporin or vancomycin), and frequent monitoring for signs of infection with early intervention are the best means to prevent infectious complications. If anything more than a superficial wound infection does arise, such as deep infection of the pump pocket or catheter, then immediate removal, culturing, and initiation of antibiotic therapy must ensue. Occasional salvage of infected pumps has been attempted in critical cases using gentamicin impregnated collagen sheet implants [8]

If an epidural abscess is suspected, an MRI with and without gadolinium should be obtained followed by intravenous antibiotics.

While chronic pain often exists in a continuous state, the intensity always varies; therefore, the goal of patient activated delivery also known as a personal therapy manager (PTM) with IDDSs is to better control this variability. PTM is usually programmed to allow the patient to receive a percentage of their total daily dose $(5 \%-20 \%)$ with activation via a remote that is programmed with a lockout interval and allowance of a maximum number of daily boluses. The addition of PTM in a patient already receiving a simple continuous infusion significantly reduces patients' overall pain scores, opioid related side effects, and consumption of concomitant oral analgesics [10].
It also increases patient satisfaction and quality of life. The vast majority of complications related to PTM usage involve issues with a malfunctioning remote. Other AEs usually result from inadvertent overdosing that can result in respiratory depression, motor block, and/or hypotension. One of the current topics of interest in the field of IT therapy is the concept of "microdosing." Microdosing describes the practice of weaning patients off of their oral opioids prior to IDDS implantation in order to use very low doses of IT opioids [1].

The differentiating features for microdosing from typical IT dosing include reducing the total IT opioid dose and its rate of escalation as well as oral opioid consumption. The 2 published studies by Grider et al and Hamza et al reported sustained pain relief with lower starting doses of opioids and limited IT opioid dose escalation without the need for oral opioid supplementation [11].

However, management of IT pump patients is practitioner dependent and variations in modalities of practice may affect parameters such as frequency and rate of dose escalation and whether or not patients are allowed to consume oral opioids. Furthermore, many patients, especially those with cancer related pain, may not tolerate complete weaning from their oral/transdermal opioids; it is unclear whether patients who are able to completely wean off opioids would do better with an IDDS delivering low dose opioids or by being managed conservatively without an implant [1].

\section{Conclusion}

Optimal use of IT therapy depends on a thorough understanding of intrathecal physiology and anatomy as well appreciation of physicochemical properties of administered medications and the mode of IT delivery.

\section{References}

[1] S. M. Hayek and M. C. Hanes, "Intrathecal therapy for chronic pain: current trends and future needs," Curr. Pain Headache Rep, Vol. 18(1), p. 388, 2014.

[2] T. L Yaksh, C. J Fisher, T. M Hockman, and A. $\mathrm{J}$ Wiese, "Current and future issues in the development of spinal agents for the management of pain," Curr. Neuropharmacol., Vol. 15(2), PP. 232-259, 2017.

[3] R. D. Penn and J. A. Paice, "Chronic intrathecal morphine for intractable pain," J. Neurosurg., Vol. 67(2), PP. 182-186, 1987.

[4] R. Rauck et al., "Long-term follow-up of a novel implantable programmable infusion pump," Neuromodulation Technol. Neural Interface, Vol. 16(2), PP. 163-167, 2013.

[5] J. Prager and M. Jacobs, "Evaluation of patients for implantable pain modalities: medical and behavioral assessment," Clin. J. Pain, Vol. 17( 3), PP. 206-214, 2001. 
[6] V. L. Ghafoor, M. Epshteyn, G. H. Carlson, D. M. Terhaar, O. Charry, and P. K. Phelps, "Intrathecal drug therapy for long-term pain management," Am. J. Heal. Pharm., Vol. 64(23), PP. 2447-2461, 2007.

[7] A. D. Kaye and R. V Shah, Case studies in pain management. Cambridge University Press, Vol. 2(11), PP. 120-135, 2014.

[8] I. E. Veizi, S. M. Hayek, S. Narouze, J. E. Pope, and N. Mekhail, "Combination of intrathecal opioids with bupivacaine attenuates opioid dose escalation in chronic noncancer pain patients," Pain Med., Vol. 12(10), PP. 1481-1489, 2011.

[9] S. R. Savage, K. L. Kirsh, and S. D. Passik, "Challenges in using opioids to treat pain in persons with substance use disorders," Addict. Sci. Clin. Pract., Vol. 4(2), p. 4, 2008.

[10] W. Ilias, B. Le Polain, E. Buchser, L. Demartini, and oPTiMa S. Group, "PatientControlled Analgesia in Chronic Pain Patients: Experience with a New Device Designed to be Used with Implanted Programable Pumps," Pain Pract., Vol. 8(3), PP. 164-170, 2008.

[11] J. S. Grider, M. E. Harned, and M. A. Etscheidt, "Patient selection and outcomes using a low-dose intrathecal opioid trialing method for chronic nonmalignant pain," Pain Physician, Vol. 14(4), PP. 343-351, 2011. 